\title{
Renormalization Group Approach to Generalized Cosmological models
}

\author{
J. Ibáñez ${ }^{1}$, S. Jhingan ${ }^{2,3}$ \\ 1 Dpto. de Física Teórica, Universidad del País Vasco, Apartado 644, 48080, Bilbao, Spain \\ 2 Centre for Theoretical Physics, Jamia Millia Islamia, Jamia Nagar, Delhi-110092, India and \\ 3 Department of Physics, Jamia Millia Islamia, Jamia Nagar, Delhi-110092, India
}

(Dated: January 31, 2018)

\begin{abstract}
We revisit here the problem of generalized cosmology using renormalization group approach. A complete analysis of these cosmologies, where specific models appear as asymptotic fixed-points, is given here along with their linearized stability analysis.

PACS numbers: 98.80.Jk, 64.60.Ak
\end{abstract}

\section{INTRODUCTION}

It is widely accepted that Einstein's general theory of relativity is an effective four-dimensional theory at lower energies and that it needs modifications at high energies. In the cosmological context, this break down of the theory results in a modification of the Friedmann equation with important consequences in the dynamical evolution at early times (see for example, [1, 2]). There has been several proposals for cosmological scenarios mostly inspired by brane-world models or by the presence of dark energy. All of them lead to modifications to the total energy density dependence of the Friedmann equation. Also, there have been studies using dynamical system approach, bringing together several of such corrections to the Friedmann equation, in order to study the conditions which lead to attractor scaling solutions [3].

In the dark energy scenarios, the energy density of the scalar field, responsible for the dark energy, is important only at late times and can account for the acceleration of the universe [4, 5, 6, 7, 8, 9, 10, 11, 12, 13, 14, 15, 16, 17, 18, 19, 20, 21, 22, 23]. Therefore, scaling solutions (i.e. solutions for which the energy density of the scalar field and the perfect fluid scale in the same way), which appear as late time attractors of the system of the evolution equations can play an important role in modeling dark energy scenarios [24]. The existence and behavior of these models in the scaling regime depends on the proposed modifications to the Friedmann equation, as well as on the form of the scalar field potential.

In this paper we analyze asymptotic evolution of modified cosmologies with a scalar field and a barotropic perfect fluid. The modification of the standard cosmology is parameterized by a function of the total energy density, in the line of Copeland et al. [3]. The analysis of the asymptotic behavior of the equations is performed using the techniques of the renormalization group ( $\mathrm{RG}$ ), which has emerged as a powerful method for doing global and asymptotic analysis of ordinary and partial differential equations (Illinois group [25], Bricmont et al [26], Caginalp [27], Moise and Ziane [28], for a reformulation of the RG approach in terms of the classical theory of envelops see Kunihiro, [29], the theory of perturbations of an isotropic universe with dynamically evolving Newton constant and Cosmologial constant [30, 31], and [32, 33] for application of RG techniques to General Relativity). We shall show in this paper that the RG technique provides a very effective method for obtaining and analyzing such solutions.

The plan of the paper is the following: In section II we describe the basic equations governing generalized cosmology. This is followed by a section where we give a very brief introduction to the RG method and apply this technique to the generalized cosmological models. Next section deals with a study of the exact scaling solutions, which are the fixed-points of the RG transformations. The stability of these exact solutions under linearized perturbations is analyzed in section V. We conclude with a brief summary.

\section{GENERALIZED COSMOLOGY}

Extending their earlier analysis of FLRW models using dynamical system approach [7], Copeland et al. showed that the modified cosmological models, such as Randall-Sundrum, Shatnov-Shani, etc. can be recovered as scaling solutions by modifying the Friedmann equation [3],

$$
H^{2}=\frac{8 \pi}{3 m_{4}^{2}} \rho G^{2}(\rho) .
$$

For $G(\rho)=$ constant, we recover the standard FLRW cosmology. Here $\rho$ is total energy density, and $m_{4}$ is the four-dimensional Planck mass. 
The model of universe considered here contains a barotropic fluid $p_{\gamma}=(\gamma-1) \rho_{\gamma}$, with adiabatic index $0 \leq \gamma \leq 2$, and a scalar field with energy density

$$
\rho_{\phi}=\frac{1}{2} \dot{\phi}^{2}+V(\phi)
$$

where $V(\phi)$ is the scalar field potential and $\rho_{\gamma}+\rho_{\phi}=\rho$. From the Einstein equations we get the following system of equations

$$
\begin{aligned}
\dot{H} & =-\frac{4 \pi G}{m_{4}^{2}}\left(G+2 \rho \frac{d G}{d \rho}\right)\left[\left(1-\frac{\gamma}{2}\right) \dot{\phi}^{2}+\gamma(\rho-V)\right] \\
\dot{\rho} & =-3 H\left[\left(1-\frac{\gamma}{2}\right) \dot{\phi}^{2}+\gamma(\rho-V)\right] \\
\dot{\rho}_{\gamma} & =-3 \gamma H \rho_{\gamma} \\
\ddot{\phi} & =-3 H \dot{\phi}-\frac{d V}{d \phi} .
\end{aligned}
$$

The evolution of scalar potential $V$ and the generalization function $G$ depends on their respective functional dependence on $\phi$, and $\rho$,

$$
\dot{V}=\left(\frac{d V}{d \phi}\right) \dot{\phi}, \quad \dot{G}=\left(\frac{d G}{d \rho}\right) \dot{\rho},
$$

and which are for the moment left arbitrary. The generalization function $G$ modifies the Hubble parameter, changing the cosmological expansion/contraction rate $(H=\dot{a} / a)$, and that correspondingly alters the evolution of all the physical variables in the system.

Before applying the RG procedure to the above set of evolution equations some comments on scaling solutions are in order. It is well known that the scalar fields with exponential potential admit scaling solutions. The form of the potential changes in the case of generalized cosmologies; Copeland et al. in ref. [3] gave a necessary and sufficient condition relating the scalar field potential and the generalization function $G$ in order to get scaling solutions. Instead of repeating their procedure to get the required condition, we give below an alternative derivation to obtain an equivalent condition.

Let's introduce a new variable

$$
x=a^{-3 \gamma}
$$

Essentially we want to use the scale factor $a(t)$, which is related to Hubble parameter, instead of the usual coordinate time $t$, as our evolution parameter. In the equation set (2.3), the equation giving evolution of the energy density of the fluid can be integrated trivially in the new parametrization as

$$
\rho_{\gamma}=\rho_{o \gamma} x
$$

where $\rho_{o \gamma}$ is an integration constant. The argument leading to the desired relation between $V$ and $G$ is now straight forward. Restricting ourselves to scaling solutions, i.e. both the scalar field density and the fluid density scale in the same way, we have

$$
\rho_{\phi} \sim \rho_{\gamma} \Rightarrow \rho_{\phi}=\rho_{o \phi} x \Rightarrow \rho=\rho_{o} x
$$

where $\rho_{o}=\rho_{o \phi}+\rho_{o \gamma}$. Using the evolution equation of the total density along with the scaling condition above, the form of potential can now be determined as

$$
V=\frac{(2-\gamma)}{2} \rho_{o \phi} x .
$$

Also, using definition of $\rho_{\phi}$, the kinetic energy term for the scalar field can be recovered as,

$$
\dot{\phi}^{2}=\gamma \rho_{o \phi} x
$$

On the other hand, we have

$$
\dot{\phi}=-3 \gamma x H \frac{d \phi}{d x}
$$


Taking the square of the above equation, substituting (2.1) and (2.7), and bearing in mind that now function $G$ depends on $x$, we get

$$
3 \sqrt{\frac{8 \pi}{3 m_{4}^{2}}} \sqrt{\frac{\gamma \rho_{o}}{\rho_{o \phi}}} \quad \phi= \pm \int \frac{d x}{x G(x)} .
$$

Once the function $G$ is fixed, the above equation gives $\phi$ as a function of $x$. Now taking the inverse of this function and substituting it in (2.6) the form of potential corresponding to the specific choice of $G$ is obtained. The condition given above is equivalent to the one obtained by Copeland et al. [3]. Therefore, condition for the scaling solution, relating generalization parameter $G$ with potential $V$, can be easily recovered starting with the scaling ansatz, that is, both the scalar field component and the fluid component scale in the same way.

\section{RENORMALIZATION GROUP APPROACH}

The renormalization group (RG) approach developed by Wilson (see for example, Wilson and Kogut [34]) has enjoyed tremendous success, and is seen as a broad philosophy rather than just a mathematical technique. In the past decade this philosophy is also applied with considerable success to understanding some very basic aspects of non-linear differential equations. In particular, equivalence of RG and intermediate asymptotics was shown by Goldenfeld et. al. (see for example, 25]). They showed that the so called anomalous dimension in RG theory are actually the non-trivial exponents appearing in the intermediate asymptotics.

The scaling solutions which we are concerned with are exact solutions, and which exhibit self-similarity at an asymptotic fixed-point. Therefore, RG seems to be a natural choice for our purpose. The form in which RG is used here was developed by Bricmont and Kupiainen [26]. The basic idea is as follows: We do a finite time integration followed by a rescaling of variables in the problem. This scaling transformation together with evolution equations gives us the RG equations. And, the fixed-points of these equations are actually the scale-invariant solutions to differential (evolution) equations which we are interested in.

We will apply now the RG method to the system of equations governing the generalized cosmology, given in the previous section. Our interest here is in the early or late time regime, where solution asymptotes to the form

$$
\lim _{t \rightarrow \infty} u(t)=t^{\chi} u^{*}(1)
$$

Here $u(t)$, refers to any variable of the former system of equations, namely $H, \rho, \phi, V, G$. The value "one" in the argument of $u^{*}(1)$, signifies the initial value of the $u$, and $\chi$ is the scaling exponent to be determined later. It is convenient to choose $t=1$ as initial time. The RG method gives us a systematic procedure to determine the exponent $\chi$, and $u^{*}(1)$, as we have illustrated below for the case of generalized cosmological models. Apart from the large time decay, this RG procedure is also applicable to the finite time decay or blow up of solutions, where self-similarity is exhibited at the asymptotic fixed-point.

Let us consider following scale transformations

$$
\begin{array}{r}
t \rightarrow L t, \quad H \rightarrow L^{e} H(L t) \equiv H_{L}, \quad \rho \rightarrow L^{a} \rho(L t) \equiv \rho_{L} \\
\dot{\phi} \rightarrow L^{b} \dot{\phi}(L t) \equiv \dot{\phi}_{L}, \quad V \rightarrow L^{c} V \equiv V_{L}, \quad G \rightarrow L^{d} G \equiv G_{L}
\end{array}
$$

We use here a number $L>1$, as a parameter for scale transformations, and the quantities subscripted with index " $L$ " are scaled quantities. The RG transformation $\mathcal{R}_{L}$ is defined as a map from one initial data set to another [32],

$$
\mathcal{R}_{L} u(x, 1)=u_{L}(x, 1) .
$$

These transformations have the semi-group property $\mathcal{R}_{L^{n}}=\mathcal{R}_{L^{n-1}} \circ \mathcal{R}_{L}$. Since the scaled quantities satisfy the original system of equations, this fixes the following exponents

$$
e=1, a=2 b=c, d=1-\frac{a}{2} \text {. }
$$

Moreover, letting $t=1$ and $L=t$, now we can express solution at arbitrary time in terms of initial values

$$
H(t)=t^{-1} H_{L}(1), \rho(t)=t^{-a} \rho_{L}(1), \dot{\phi}(t)=t^{-\frac{a}{2}} \dot{\phi}_{L}(1), V(t)=t^{-a} V_{L}, G(t)=t^{\frac{a}{2}-1} G_{L}
$$

where quantities sub-scripted with $L$ are constants. The exponent $a$ is the anomalous dimension: the field equations alone do not fix it and it should be determined by initial or boundary conditions. For instance, when one chooses a particular model by fixing the function $G$, parameter $a$ gets determined. 
Note from Eq. (3.4) that the evolution of $\phi$ (as well as $\dot{\phi}$ ) depends on the value of parameter $a$. This behavior, as we will see later, has consequences on the nature of the potential in the scaling regime. In particular it is the parameter $a$, which distinguishes FRW standard cosmology from other "generalized" models.

Comments are now in order about nature of the potential $V$. Though the functional form of the potential is left free, clearly in the scaling regime it gets fixed, Eq. (3.4). Moreover, the functional form of $G$ also is not arbitrary. As argued in the previous paragraph, depending on the value of parameter $a$, the scaling regime can be divided in two categories; $a=2$, which gives the usual FLRW models and $a \neq 2$, the generalized cosmology.

$\underline{\mathbf{a}=\mathbf{2}}$ : From Eq. (3.4), we get

$$
V(\phi)=V_{0} \exp \left(-2 \phi / \dot{\phi}_{L}\right), \quad G(\rho)=G_{0}
$$

Where $V_{0}=V_{L}(1)$ and $G_{0}=G_{L}(1)$ are constants. Therefore, in the scaling regime we have a exponential potential and the generalization function $G$ is a constant, i.e., FLRW cosmology.

$\underline{\mathbf{a} \neq \mathbf{2}}$ : In this case $G$ and $V$ have a power law dependence on $\rho$ and $\phi$, respectively, in the scaling regime

$$
V(\phi)=V_{0} \phi_{L}^{\frac{2 a}{a-2}}, \quad G(\rho)=G_{0} \rho_{L}^{\frac{2-a}{2 a}},
$$

where $G_{0}$ and $V_{0}$ have simple dependence on the $L$ sub-scripted quantities.

We draw a comparison now between the scaling solutions in cosmology, discussed in the previous section, and the scale invariant solutions which arise as asymptotic fixed-points of the RG analysis. First note that we are using as a variable the total energy density $\rho$, instead of the density of the perfect fluid $\rho_{\gamma}$. However, from Eq. (2.3) we have the following scaling relation for the perfect fluid energy density $\rho_{\gamma} \sim t^{-a}$. It is easy now to see that the energy density of the perfect fluid and the energy density of the scalar field scale in the same way

$$
\frac{\rho_{\phi}}{\rho_{\gamma}}=\frac{{\dot{\phi_{L}}}^{2}+2 V_{L}}{2 \rho_{\gamma_{L}}}=\text { constant }
$$

which is the usual definition of scaling solutions in scalar field cosmologies. Therefore, in RG method we recover all the scaling solutions of the system as a subset of scale invariant solutions, provided that both components of energy density are non-vanishing.

The large $L$ in our notation means late time. Therefore, applying the RG transformation repeatedly we can recover the long time behavior of the solution. Defining a auxiliary parameter $\tau$ through $L=\exp (\tau)$, the RG equations are

$$
\begin{gathered}
\frac{d H_{L}}{d \tau}=H_{L}+\left.\frac{d H_{L}}{d t}\right|_{t=1}=H_{L}-\left\{\frac{4 \pi G_{L}}{m_{4}^{2}}\left(G_{L}+2 \rho_{L} \frac{d G_{L}}{d \rho_{L}}\right)\left[\left(1-\frac{\gamma}{2}\right) \dot{\phi}_{L}^{2}+\gamma\left(\rho_{L}-V_{L}\right)\right]\right\}_{t=1} \\
\frac{d \rho_{L}}{d \tau}=a \rho_{L}+\left.\frac{d \rho_{L}}{d t}\right|_{t=1}=a \rho_{L}-\left\{3 H_{L}\left[\left(1-\frac{\gamma}{2}\right) \dot{\phi}_{L}^{2}+\gamma\left(\rho_{L}-V_{L}\right)\right]\right\}_{t=1} \\
\frac{d \dot{\phi}_{L}}{d \tau}=\frac{a}{2} \dot{\phi}_{L}+\left.\frac{d \dot{\phi}_{L}}{d t}\right|_{t=1}=\frac{a}{2} \dot{\phi}_{L}-\left\{3 H_{L} \dot{\phi}_{L}-\frac{d V_{L}}{d \phi_{L}}\right\}_{t=1} \\
\frac{d V_{L}}{d \tau}=a V_{L}+\left.\frac{d V_{L}}{d t}\right|_{t=1}=a V_{L}+\left\{\left(\frac{d V_{L}}{d \phi_{L}}\right) \dot{\phi}_{L}\right\}_{t=1} \\
\frac{d G_{L}}{d \tau}=\left(1-\frac{a}{2}\right) G_{L}+\left.\frac{d G_{L}}{d t}\right|_{t=1}=\left(1-\frac{a}{2}\right) G_{L}+\left\{\left(\frac{d G_{L}}{d \rho_{L}}\right) \dot{\rho}_{L}\right\}_{t=1} .
\end{gathered}
$$

Which is a set of algebraic equations since the quantities on the right hand side are all evaluated at $t=1$. Note that the system of equations above has no dependence on $L$; this is expected since the system (2.3) is scale invariant. 


\section{SCALE INVARIANT SOLUTIONS}

The scale invariant solutions appear as fixed-points to the RG equations. The fixed-points are those points which are mapped onto themselves by the RG transformations for any $L>1$

$$
\mathcal{R}_{L} u^{*}=u^{*}
$$

and which is equivalent to

$$
\frac{d u^{*}}{d \tau}=0
$$

Therefore, the equations determining the fixed-points of our system are

$$
\frac{d H_{L}^{*}}{d \tau}=0, \quad \frac{d \rho_{L}^{*}}{d \tau}=0, \quad \frac{d \dot{\phi}_{L}^{*}}{d \tau}=0, \quad \frac{d V_{L}^{*}}{d \tau}=0
$$

As discussed earlier in this paper, if classified in terms of the potential function of the scalar field there are two classes of solutions corresponding to the exponential and the power-law potentials; equivalently the system can also be classified in terms of the generalization function $G$. Moreover, these two different classes also differ in their value of anomalous dimension " $a$ " and which, therefore, can also serve as an alternative way of classifying these fixed-points.

\section{A. Exponential Potential $(a=2)$}

To facilitate a comparison with earlier works in FLRW scalar field cosmology we shall use $\rho_{\gamma}$, as a variable in this subsection instead of the total energy density $\rho$. Moreover, we work with the notation used in [7], defining $\kappa=8 \pi G$. The set of RG equations simplifies to

$$
\begin{gathered}
\frac{d H_{L}}{d \tau}=H_{L}-\left\{\frac{\kappa^{2}}{2}\left(\gamma \rho_{\gamma L}+\dot{\phi}_{L}^{2}\right)\right\}_{t=1} \\
\frac{d \rho_{\gamma L}}{d \tau}=2 \rho_{\gamma L}-3 \gamma\left\{\rho_{\gamma L} H_{L}\right\}_{t=1} \\
\frac{d \dot{\phi_{L}}}{d \tau}=\dot{\phi_{L}}-3\left\{\dot{\phi_{L}} H_{L}-\frac{d V_{L}}{d \phi_{L}}\right\}_{t=1}
\end{gathered}
$$

Therefore, the fixed-points for this system are

$$
\frac{d H_{L}^{*}}{d \tau}=0, \quad \frac{d \rho_{\gamma L}^{*}}{d \tau}=0, \quad \frac{d \dot{\phi}_{L}^{*}}{d \tau}=0 .
$$

The fixed-points should also satisfy the Friedmann equation

$$
H^{* 2}=\frac{\kappa^{2}}{3}\left(\rho_{\gamma}^{*}+\frac{1}{2} \dot{\phi}^{2}+V^{*}\right) .
$$

From the second equation in Eq. set (4.3), we see that there are two different sets of fixed-points: those with $\rho_{\gamma}^{*}=0$ and those with $\rho_{\gamma}^{*} \neq 0$. Let's start with the fixed-points characterized by $\rho_{\gamma}^{*}=0$. This case can be further subdivided into two sub-cases. In the first case, corresponding to $V^{*}=0$, we obtain

$$
V^{*}=0, \quad\left(\frac{d V}{d \phi}\right)^{*}=0, \quad H^{*}=\frac{1}{3}, \quad \rho_{\gamma}^{*}=0, \quad \dot{\phi}^{*}= \pm \frac{1}{\kappa} \sqrt{\frac{2}{3}} .
$$

This solution corresponds to a massless scalar field cosmology.

In the second case, $V^{*} \neq 0$, the nature of potential in the neighborhood of the fixed-point is given by (3.5). Comparing the potential (3.5) with the exponential potential used in [7], $V=V_{0} \exp (-\kappa \lambda \phi)$, the fixed-point is given by

$$
\dot{\phi}^{*}=\frac{2}{\kappa \lambda}, \quad \rho_{\gamma}^{*}=0, \quad H^{*}=\frac{2}{9 \lambda^{2}}, \quad V^{*}=\frac{2}{\kappa^{2} \lambda^{2}}\left(\frac{6}{\lambda^{2}}-1\right) .
$$


It is a exponential potential scalar field cosmology.

For the fixed-points with non-zero perfect fluid $\rho_{\gamma}^{*} \neq 0$ the value of Hubble parameter is determined $H^{*}=2 / 3 \gamma$. And we have following sub-cases due the nature of the potential. One case with $V^{*}=0$, and the solution for fixed-point is

$$
H^{*}=\frac{2}{3 \gamma}, \quad \rho_{\gamma}^{*}=\frac{4}{3 \kappa^{2} \gamma^{2}}, \quad \dot{\phi}^{*}=0, \quad V^{*}=0 .
$$

Which is a perfect fluid only cosmology. The second case $V^{*} \neq 0$, the fixed-point is

$$
H^{*}=\frac{2}{3 \gamma}, \quad \rho_{\gamma}^{*}=\frac{4}{\kappa^{2} \gamma^{2}} \frac{\gamma-3 \lambda^{2}}{9 \lambda^{2}}, \quad \dot{\phi}^{*}=\frac{2}{3 \kappa \lambda}, \quad V^{*}=\frac{2(2-\gamma)}{9 \kappa^{2} \gamma \lambda^{2}},
$$

for $\lambda^{2}>3 \gamma$.

The fixed-points obtained are the same as in given in 7], where the scalar field potential is assumed to be of exponential form from the very beginning, and the system of equations was written in terms of normalized variables that make the phase space of the autonomous system bounded. It is interesting to note that imposing scale invariance we obtain the same results without using normalized variables. On the other side, it is reasonable to think that, what are called scaling solutions should be scale invariance solutions, since in that case the scalar field energy density and the energy density of the matter scale as a power of the scale factor. Assuming this we have proved that the only potential that gives scale invariance solutions is of exponential type in FLRW cosmology.

\section{B. Power-law potentials $(a \neq 2)$}

In deriving the expression for potential in the scaling regime we have used the scaling behavior of $\phi$, i.e.,

$$
\frac{d \phi_{L}}{d \tau}=\left(\frac{a}{2}-1\right) \phi_{L}+\dot{\phi}_{L}
$$

implying $2 \dot{\phi}^{*}=(2-a) \phi^{*}$ at the fixed-point.

Let's note also that, as in the standard cosmology, there are two classes of fixed-points depending on whether $\rho_{\gamma}$ vanishes or not. The quantity $\rho_{\gamma}$, however, is not a variable in our system of equations (3.8) (we have used $\rho$ instead), nevertheless $\rho_{\gamma}$ satisfies the following equation

$$
\frac{d \rho_{\gamma}}{d \tau}=\rho_{\gamma}(a-3 \gamma H)
$$

which means that if fluid density is non-vanishing at the fixed point $\rho_{\gamma}^{*} \neq 0$, the Hubble parameter takes the critical value $H^{*}=a / 3 \gamma$.

To analyze the fixed-points when $a \neq 2$, we first consider $V=0$. In this case the RG equations simplify considerably

$$
\begin{gathered}
H^{* 2}=\frac{8 \pi G_{0}^{2}}{3 m_{4}^{2}} \rho^{* \frac{2}{a}} \\
2 a \rho^{*}=3 H^{*}\left[(2-\gamma) \dot{\phi}^{* 2}+2 \gamma \rho^{*}\right] \\
a \dot{\phi}^{*}=6 H^{*} \dot{\phi}^{*}
\end{gathered}
$$

giving the following set of fixed-points

$$
\begin{array}{ccc}
\dot{\phi}^{*}=0, & \rho^{*}=\left(\frac{a^{2} m_{4}^{2}}{24 \pi \gamma^{2} G_{0}^{2}}\right)^{\frac{a}{2}}, & H^{*}=\frac{a}{3 \gamma} \\
\dot{\phi}^{*}= \pm \sqrt{2 \rho^{*}}, & \rho^{*}=\left(\frac{a^{2} m_{4}^{2}}{96 \pi G_{0}^{2}}\right)^{\frac{a}{2}}, & H^{*}=\frac{a}{6}
\end{array}
$$

The fixed-point (4.10) corresponds to vanishing of the scalar field and (4.11) to the vanishing of the perfect fluid. Therefore, only one component survives in the scaling regime. Note that (4.11) is not a limiting case of (4.10) for $\gamma=2$. 
The fixed-points in the more general case with non-zero potential are governed by the following set of algebraic equations

$$
\begin{gathered}
H^{* 2}=\frac{8 \pi G_{0}^{2}}{3 m_{4}^{2}} \rho^{* \frac{2}{a}} \\
8 a \rho^{*}=3 H^{*}\left[(2-\gamma)(2-a)^{2} \phi^{* 2}+8 \gamma\left(\rho^{*}-V_{0} \phi^{* \frac{2 a}{a-2}}\right)\right] \\
H^{*} \phi^{*}=\frac{a}{6} \phi^{*}-\frac{4 a V_{0}}{3(a-2)^{2}} \phi^{* \frac{a+2}{a-2}}
\end{gathered}
$$

These cannot be solved in general however various special cases corresponding to different values of parameter $a(\neq 2)$ can be analyzed.

First we consider the simplest scenario where potential $V$ vanishes in the asymptotic regime due to vanishing of the scalar field itself $\left(\phi^{*}=0\right)$. This is unlike the previous case where potential was identically zero (due to, say, $V_{0}=0$ ) to begin with. We recover following fixed-point for vanishing of the scalar field $\phi^{*}=0$,

$$
H^{*}=\frac{a}{3 \gamma}, \rho^{*}=\left(\frac{a^{2} m_{4}^{2}}{24 \pi \gamma^{2} G_{0}^{2}}\right)^{\frac{a}{2}}, V^{*}=0
$$

This is similar to the previous case, except the fact that the range of anomalous dimension $a$ is restricted to $|a|>2$, and that the cosmology is driven towards vanishing potential which is non-zero to begin with.

The fixed-points corresponding to a non-vanishing potential in the scaling regime can be divided in two families. The one corresponding to vanishing fluid component in scaling regime is given by

$$
H^{*}= \pm \sqrt{\frac{8 \pi G_{0}^{2}}{3 m_{4}^{2}}} \rho^{* \frac{1}{a}}, \quad \rho^{*}=\left[\frac{3 \pi(2-a)^{4} G_{0}^{2}}{2 a^{2} m_{4}^{2}}\right]^{\frac{a}{2(a-1)}} \phi^{* \frac{2 a}{a-1}}, \quad V^{*}=V_{0} \phi^{* \frac{2 a}{a-2}} .
$$

The plus and the minus signs correspond to expanding and contracting models, respectively, and the $\phi^{*}$ in the equation above is given by the solution to following algebraic equation $(a \neq 1,2)$;

$$
\phi^{* \frac{4}{a-2}}-\frac{1}{V_{0}}\left(1-\frac{a}{2}\right)^{\frac{2 a}{a-1}}\left[ \pm \sqrt{\frac{24 \pi G_{0}^{2}}{a^{2} m_{4}^{2}}}\right]^{\frac{a}{(a-1)}} \phi^{* \frac{2}{a-1}}+\frac{(2-a)^{2}}{8 V_{0}}=0 .
$$

The case $a=1$ needs to be treated separately and the fixed-point is of the form

$$
H^{*}= \pm \sqrt{\frac{8 \pi G_{0}^{2}}{3 m_{4}^{2}}} \rho^{*}, \quad \rho^{*}= \pm \sqrt{\frac{3 \pi G_{0}^{2}}{2 m_{4}^{2}}}\left(\frac{m_{4}^{2}}{12 \pi G_{0}^{2}}+V_{0}\right), \quad \phi^{* 2}= \pm \sqrt{\frac{2 m_{4}^{2}}{3 \pi G_{0}^{2}}}, \quad V^{*}=V_{0} \phi^{* \frac{2 a}{a-2}}
$$

The other fix-point corresponding to non-vanishing of both the scalar field and the perfect fluid is of the form

$$
H^{*}=\frac{a}{3 \gamma}, \quad \phi^{*}=\left[\frac{(2-\gamma)(2-a)}{4 \gamma V_{0}}\right]^{\frac{a-2}{4}}, \quad \rho^{*}=\left(\frac{a^{2} m_{4}^{2}}{24 \pi \gamma^{2} G_{0}^{2}}\right)^{\frac{a}{2}}, \quad V^{*}=V_{0} \phi^{* \frac{2 a}{a-2}} .
$$

For $a=2$ system reduces to usual FLRW models with exponential potentials which were analyzed in the previous subsection.

\section{Stability}

The scaling solutions studied in the previous section arise as fixed-points of the RG equations. The stability in time evolution around a self-similar solution is stability of RG flow near the fixed-point. We do a linear perturbation analysis here, considering perturbations in the initial data space staying close to the fixed-points and searching for relevant modes, i.e., modes which make the flow diverge from the fixed-points.

Let us define following perturbation quantities

$$
H=H^{*}+\delta H, \rho=\rho^{*}+\delta \rho, \dot{\phi}=\dot{\phi}^{*}+\delta \dot{\phi}, \phi=\phi^{*}+\delta \phi, V=V^{*}+\delta V, G=G^{*}+\delta G
$$


Where all perturbed quantities are small $(\ll 1)$. The Friedmann equation can be linearized as

$$
H^{*} \delta H=\frac{4 \pi}{3 m_{4}^{2}} G^{*}\left[G^{*} \delta \rho+2 \rho^{*} \delta G\right]
$$

and can be used to relate variation in $H$ with other variables. However, this reduction in variables can make the equation set much more complicated and for the moment we will work with the full set of variables.

The linearized RG equations take the following form

$$
\begin{aligned}
\frac{d \delta \rho}{d \tau}= & \left(a-3 \gamma H^{*}\right) \delta \rho+3(\gamma-2) H^{*} \dot{\phi}^{*} \delta \dot{\phi}+3 \gamma H^{*} \delta V-\frac{3}{2}\left[(2-\gamma)\left(\dot{\phi}^{*}\right)^{2}+2 \gamma\left(\rho^{*}-V^{*}\right)\right] \delta H \\
\frac{d \delta \dot{\phi}}{d \tau}= & \left(\frac{a}{2}-3 H^{*}\right) \delta \dot{\phi}-3 \dot{\phi}^{*} \delta H+\frac{2 a}{(a-2)} \frac{V^{*}}{\left(\dot{\phi}^{*}\right)^{2}} \delta \phi+\frac{2 a}{(2-a) \dot{\phi}^{*}} \delta V \\
\frac{d \delta \phi}{d \tau}= & \left(\frac{a}{2}-1\right) \delta \phi+\delta \dot{\phi} \\
\frac{d \delta V}{d \tau}= & \frac{2 a}{(a-2)} \frac{V^{*}}{\phi^{*}} \delta \dot{\phi}-\frac{2 a}{(a-2)} \frac{V^{*} \dot{\phi}^{*}}{\left(\phi^{*}\right)^{2}} \delta \phi+a\left(1+\frac{2}{(a-2)} \frac{\dot{\phi}^{*}}{\phi^{*}}\right) \delta V \\
\frac{d \delta G}{d \tau}= & \frac{(a-2)}{2 a}\left\{-\left[\left(a-3 \gamma H^{*}\right)+\frac{3}{2} \frac{H^{*}}{\rho^{*}}\left[(\gamma-2)\left(\dot{\phi}^{*}\right)^{2}+2 \gamma V^{*}\right]\right] \delta G+\frac{3}{2} \frac{G^{*} H^{*}}{\left(\rho^{*}\right)^{2}}\left[(\gamma-2)\left(\dot{\phi}^{*}\right)^{2}\right.\right. \\
& \left.\left.+2 \gamma V^{*}\right] \delta \rho+3\left[\gamma G^{*}-\frac{1}{2} \frac{G^{*}}{\rho^{*}}\left[(\gamma-2)\left(\dot{\phi}^{*}\right)^{2}+2 \gamma V^{*}\right]\right] \delta H+3(2-\gamma) \frac{H^{*} G^{*} \dot{\phi}^{*}}{\rho^{*}} \delta \dot{\phi}\right\} .
\end{aligned}
$$

We now find the normal modes with following ansatz

$$
\delta f=\bar{f} \exp (\omega \tau)
$$

where $f$ is a representative variable for $H, \rho, \dot{\phi}, \phi, V$ and $G$, and over bar signifies quantity to be constant. With this ansatz and also simplifying system using (4.18) we can now look for any relevant modes.

Of all the fixed-points listed in the previous section the one given by (4.17) is of particular interest since this corresponds to late time regime where both scalar field as well as perfect fluid component are non-vanishing. Taking these particular fixed-point values the stability of the system depends of the following matrix

$\left(\begin{array}{c|c|c|c|c}\omega+\frac{12 \pi \gamma^{2}}{a m_{4}^{2}} G^{* 2} \rho^{*} & \frac{a(\gamma-2)(a-2)}{2 \gamma} \phi^{*} & -a & 0 & \frac{24 \pi \gamma^{2}}{a m_{4}^{2}} G^{*} \rho^{* 2} \\ \hline \frac{6 \pi \gamma(2-a)}{a m_{4}^{2}} G^{* 2} \phi^{*} & \omega+\frac{a(2-\gamma)}{2 \gamma} & \frac{a(a-2)(\gamma-2)}{4 \gamma} & \frac{2 a}{(a-2) \phi^{*}} & \frac{12 \pi \gamma(2-a)}{a m_{4}^{2}} G^{*} \rho^{*} \phi^{*} \\ \hline 0 & -1 & \omega+1-\frac{a}{2} & 0 & 0 \\ \hline 0 & \frac{a(a-2)(\gamma-2)}{4 \gamma} \phi^{*} & \frac{a(a-2)^{2}(\gamma-2)}{8 \gamma} \phi^{*} & \omega & 0 \\ \hline \frac{6 \pi \gamma^{2}(2-a)}{a^{2} m_{4}^{2}} G^{* 3} & \frac{(2-\gamma)(a-2)^{2}}{4 \gamma} \frac{G^{*} \phi^{*}}{\rho^{*}} & 0 & \frac{(a-2)}{2} \frac{G^{*}}{\rho^{*}} & \omega-\frac{12 \pi \gamma^{2}(a-2)}{a^{2} m_{4}^{2}} G^{* 2} \rho^{*}\end{array}\right)$

The values of $\omega$ are determined by vanishing of the determinant of the above matrix. The two eigenvalues are zero and the remaining three are determined by solution of a cubic equation

$$
\begin{gathered}
\omega^{3}+\left[\left(1-a+\frac{a}{\gamma}\right)+\frac{24 \pi \gamma^{2}}{a^{2} m_{4}^{2}} G^{* 2} \rho^{*}\right] \omega^{2}+\left[\frac{a(2-\gamma)}{\gamma}+\frac{6 \pi(\gamma-2)(a-2)^{2}}{a m_{4}^{2}} G^{* 2} \phi^{* 2}\right. \\
\left.+\frac{24 \pi \gamma(\gamma+a-\gamma a)}{a^{2} m_{4}^{2}} \rho^{*}\right] \omega+\frac{6 \pi(\gamma-2)}{a m_{4}^{2}}\left[(a-2)^{2} \phi^{* 2}+4 \gamma \rho^{*}\right] G^{* 2}=0
\end{gathered}
$$

Solutions of this equation and therefore criterion for stability of the fixed-points depends on the value of the anomalous dimension $a$ and and other constants in the problem.

\section{SUMMARY}

We have analyzed in this paper long time behavior of the cosmological equations By using the Renormalization Group method, paying special attention to the scaling solutions in two different cases. The first case of standard 
FRLW cosmology has been widely studied and it serves us to illustrate the method we use. Although the system in this simple case can be analyzed by means of dynamical system technique, this analysis depends upon the choice of normalized variables which are not easy to find. The RG method avoids this problem: the scale invariance solutions found describe all the scaling solutions.

We also applied RG method to the generalized cosmologies, which imply modifications of the Friedmann equation. The RG equations give the scaling solutions of the system and we have shown that in the scaling regime the potential of the scalar field and the function which describes the modifications to the Friedmann equation have a power law dependence on their respective variables.

The RG method is a simple but powerful tool to investigate scaling solutions for extended cosmologies. We have proved that to get scaling solutions, during the dynamical evolution, the scalar field potential should be either power law or exponential and the function G, that generalizes Friedmann equation, should be a power law of the total density. Only the fixed point given by Eq. (4.17) represents a scaling solution, whereas the rest of fixed points give cosmologies with either vanishing fluid or vanishing scalar field. The stability of the scaling solution fixed point is given by Eq. (4.20), and it depends on the particular model under study through the constants appearing in that equation. However, the most relevant of these constants is the anomalous dimension " $a$ ". Finally, it would be interesting to study the evolution of a larger variety of field models as K-essence, phantom, quintaessence, etc. (see [35] for a review) with this technique.

\section{Acknowledgements}

We would like to acknowledge useful discussions with Takahiro Tanaka and participants of the JGRG14, and YKIS 2005, held in Kyoto. SJ acknowledges support under a JSPS fellowship, and JI acknowledges financial support under grant FIS 2004-01626.

\section{References}

[1] R. Maartens, Phys. Rev. D 62 (2000) 084023.

[2] A. A. Coley, Phys. Rev. D 66 (2002) 023512.

[3] E. J. Copeland, S. Lee, J. E. Lidsey and S. Mizuno, Phys. Rev. D 71 (2005) 023526.

[4] B. Ratra and P. J. E. Peebles, Phys. Rev. D 37 (1988) 3406.

[5] R. R. Caldwell, R. Dave and P. J. Steinhardt, Phys. Rev. Lett. 80 (1998) 1582; I. Zlatev, L. M. Wang and P. J. Steinhardt, Phys. Rev. Lett. 82 (1999) 896; P. J. Steinhardt, L. M. Wang and I. Zlatev, Phys. Rev. D 59 (1999) 123504.

6] C. Wetterich, Nucl. Phys. B302 (1988) 668.

[7] E. J. Copeland, A. R. Liddle and D. Wands, Phys. Rev. D 57 (1998) 4686.

[8] P. G. Ferreira and M. Joyce, Phys. Rev. D 58 (1998) 023503.

[9] A. R. Liddle and R. J. Scherrer, Phys. Rev. D 59 (1999) 023509.

[10] T. Barreiro, B. de Carlos and E. J. Copeland, Phys. Rev. D 58 (1998) 083513.

[11] G. Huey, P. J. Steinhardt, B. A. Ovrut and D. Waldram, Phys. Lett. B 476 (2000) 379.

[12] T. Barreiro, B. de Carlos and N. J. Nunes, Phys. Lett. B 497 (2001) 136.

[13] V. Sahni and A. Starobinsky, Int. J. Mod. Phys. D 9 (2000) 373.

[14] A. de la Macorra and G. Piccinelli, Phys. Rev. D 61 (2000) 123503.

[15] S. C. C. Ng, N. J. Nunes and F. Rosati, Phys. Rev. D 64 (2001) 083510.

[16] E. J. Copeland, A. R. Liddle and J. E. Lidsey, Phys. Rev. D 64 (2001) 023509.

[17] G. Huey and J. E. Lidsey, Phys. Lett. B 514 (2001) 217.

[18] V. Sahni, M. Sami and T. Souradeep, Phys. Rev. D 65 (2002) 023518; M. Sami and V. Sahni, arXiv:hep-th/0402086

[19] G. Huey and R. K. Tavakol, Phys. Rev. D 65 (2002) 043504.

[20] A. S. Majumdar, Phys. Rev. D 64 (2001) 083503.

[21] N. J. Nunes and E. J. Copeland, Phys. Rev. D 66 (2002) 043524.

[22] R. J. van den Hoogen, A. A. Coley and Y. He, Phys. Rev. D 68 (2003) 023502; R. J. van den Hoogen and J. Ibanez, Phys. Rev. D 67 (2003) 083510.

[23] J. E. Lidsey and N. J. Nunes, Phys. Rev. D 67 (2003) 103510.

[24] S. Tsujikawa and A. R. Liddle, JCAP 0403 (2004) 001.

[25] N. Goldenfeld, O. Martin and Y. Oono, J. Sci. Comput. 4 (1989) 355; L. Y. Chen, N. Goldenfeld and Y. Oono, Phys. Rev. Lett. 73 (1994) 1311; L. Y. Chen, N. Goldenfeld and Y. Oono, Phys. Rev. E, 54 (1996) 376.

[26] J. Bricmont, A. Kupiainen and G. Lin, Commun. Pure and Appl. Math. XLVII (1994) 893. 
[27] G. Caginalp, Phys. Rev. E, 53 (1996) 66; G. Caginalp, Nonlinearity 10 (1997) 1217; H. Merdan and G. Caginalp, Apl. Math. Lett. 17 (2004) 217.

[28] I. Moise and M. Ziane, J. Dyn. Diff. Eqn. 13 (2001) 275.

[29] T. Kunihiro, Prog. Theor. Phys. 94 (1995) 503; S. Ei, K. Fujii and T. Kunihiro, Ann. Phys. 280 (2000) 236.

[30] A. Bonnano and M. Reuter, Phys.Lett.B 527 (2002) 9 ; M. Reuter and H. Weyer, Phys. Rev. D 69 (2004) 104022 ; A. Bonnano and M. Reuter, Int. J. Mod. Phys. D 13 (2004) 107.

[31] I. L. Shapiro, J. Sola, C. Espana-Bonet, and P. Ruiz-Lapuente, Phys. Lett. B 574 (2003) 149.

[32] T. Koike, T. Hara and S. Adachi, Phys. Rev. Lett. 74 (1995) 5170; O. Iguchi, A. Hosoya and T. Koike, Phys. Rev. D 57 (1998) 3340 .

[33] J. Ibáñez and S. Jhingan, Phys. Rev. D 70 (2004) 063507.

[34] K. G. Wilson and J. Kogut, Phys. Rep. 12 (1974) 75.

[35] E. J. Copeland, M. Sami and S. Tsujikawa, hep-th/0603057 